\title{
Article
}

Mycosphere

\section{Luminescence of wood samples during long-term storage}

\section{Puzyr AP and Medvedeva SE}

Institute of Biophysics, Siberian Branch of Russian Academy of Science, Federal Research Center "Krasnoyarsk Science Center SB RAS", Akademgorodok, 660036 Krasnoyarsk, Russia

Puzyr AP, Medvedeva SE 2016 - Luminescence of wood samples during long-term storage. Mycosphere 7 (6), 716-727, Doi 10.5943/mycosphere/7/6/2

\begin{abstract}
The present study describes changes in the mycelium of the fungus growing on the luminescent wood collected on Borneo Island in early December 2013 that occurred during 31 months of storage. The study shows that wood samples retain their ability to emit light, forming two types of luminescent mycelium: surface mycelium and aerial mycelium. The hyphae of the surface mycelium form on the surface of the wood sample and then spread over the surface of the polyethylene bag or over the surface of the bottom of tissue culture flasks containing the samples. The aerial mycelium develops later and only in tissue culture flasks, forming biomass composed of local interlaced hyphae, growing upward. The surface mycelium is characterized by non-uniform "flickering" luminescence along the hyphae. There is no diurnal periodicity in the luminescence of this fungus, but luminescence is increased by mechanical disturbance or exposure to ultraviolet radiation. The local impact of these factors causes an increase in luminescence of the mycelium regions that have not been directly affected. It has been assumed that the variable level of luminescence is an individual trait of this fungus species. The results obtained in this study suggest that luminescent wood found on Borneo Island contains mycelium of a fungus species, whose luminescent properties are essentially different from those of the fungi described in the scientific literature.
\end{abstract}

Key words - light emitting wood - luminous mycelia

\section{Introduction}

Data on luminescence of the pure culture of mycelium isolated from light-emitting basidiomycetes have been extensively reported in the literature. Such cultures were grown on various nutrient media and used as bioassays (Bermudes et al. 1990, Weitz et al. 2001, 2002, Horswell et al. 2006, Paton et al. 2006, Mendes et al. 2008, 2010, Vydryakova et al. 2011). One of the ways to maintain the culture is to transfer biomass to distilled water (Bilai 1982), and soaking mycelium in water causes an increase in luminescence (Mori et al. 2011, Bondar et al. 2012, 2013).

It has been proved that fungal luciferase is associated with membrane-rich fractions (Airth \& Foerster 1962, Airth et al. 1966, Oliveira \& Stevani 2009). However, other data reported by different groups of researchers may be contradictory. For instance, some of them state that extracts from non luminescent species did not result in light emission when cross-reacted (Oliveira et al. 2012) while others prove that extracts from non luminescent species did result in light emission when cross-reacted (Purtov et al. 2015, Puzyr et al. 2016). 
Some authors reported that laboratory cultures of certain fungi showed circadian rhythms (Berliner 1961, Kamzolkina 1982, Bermudes et al. 1990, Oliveira et al. 2015). However, other researchers did not observe any no circadian rhythms in the fungi (Weitz et al. 2001, Deheyn \& Latz 2007, Mihail \& Bruhn 2007).

The possibility of opening new species of glowing mushrooms in our time it has been shown (Desjardin et al. 2010, Capelari et al. 2011, Chew et al. 2014). At the same time, only two papers describe investigations of the mycelium of luminescent fungi contained in light-emitting wood. Sabharwal et al. (1983) reported the characteristics of light emission in vivo for different samples of the shining wood collected from different trees. That study suggested that the emission spectrum of the light from the wood is around $518 \mathrm{~nm}$ and water is not only important for emission but has a function in the kinetics of the reaction. Deheyn and Latz (2007) described a laboratory study of the emission spectrum of fluorescence, effect of hydration on bioluminescence, effect of temperature on bioluminescence, and day - night variations in bioluminescence of luminous pieces of wood. The authors reported bioluminescence characteristics of natural wood samples: the light was yellow, light production was a continuous phenomenon that did not vary between day and night. Bioluminescence intensity was affected by temperature change, and bioluminescence of fungus increased upon exposure to water. The authors believe that «This study is unique in reporting bioluminescence characteristics of a freshly collected luminous fungus on its natural wood substrate, as opposed to fungus cultured in the laboratory on plates».

In our previous work, we investigated pure culture of the fungus isolated from the lightemitting wood found on Borneo Island. The culture is registered in the IBP Culture Collection (CCIBSO 836) as IBSO 2371. We also reported that under moist non sterile conditions, mycelium contained in the wood was capable of emitting light and producing new mycelium for 22 months (Puzyr et al. 2016).

In this study, we investigated luminescence of fungal mycelium growing on wood in laboratory for 31 months and the effects of external factors on luminescence.

\section{Materials \& Methods}

Samples of luminescent wood collected on Borneo Island in early December 2013 had been kept in a thermostatic chamber (TSO-1/80, SPU, Russia) at a temperature of $27^{\circ} \mathrm{C}$ until the beginning of the experiment (July 2016). In this study, we used samples that differed in their size and storage conditions. Large-sized samples were placed into two, 1300 and $650 \mathrm{ml}$, tissue culture flasks (Techno Plastic Products, Switzerland). Each tissue culture flask contained cotton wool as a water-absorbing layer and distilled water. Small-sized samples were placed into polyethylene (PE)bags with zip-lock, containing water. All manipulations - sample collection, transportation, and placement into tissue culture flasks and PE bags as well as addition of water - were conducted under non sterile conditions. Tissue culture flasks were hermetically sealed, and neither air nor water had been changed. Although the PE bags had zip locks, water evaporation occurred. Therefore, we had to add water periodically, also letting the air into the bags.

The magnitude and dynamics of the change in the light signal were monitored by using a Glomax® 20/20 luminometer (Promega Bio Systems Sunnyvale, Inc., U.S.). During storage, changes visually observed in the samples were recorded with a GelDoc XR Imaging System (BioRad Laboratories, Inc., U.S.). Color photographs were taken with a Canon EOS 6D camera. The conditions for detecting the luminescent signal and visible light images of the samples with daylight lamps off were the same as described elsewhere (Puzyr et al. 2016). A constant temperature of $26^{\circ} \mathrm{C}$ was maintained in the laboratory with a BALLU BSC-12H air conditioner (Ballu Industrial Group, China). Samples were irradiated with Sankyo Denki G8T5 lamps emitting ultraviolet radiation (Japan) in Universal Hood II and a $395 \mathrm{~nm}$ ultraviolet flashlight (Ultrafire WF502B UV). Irradiation was performed for $10 \mathrm{~s}$. 

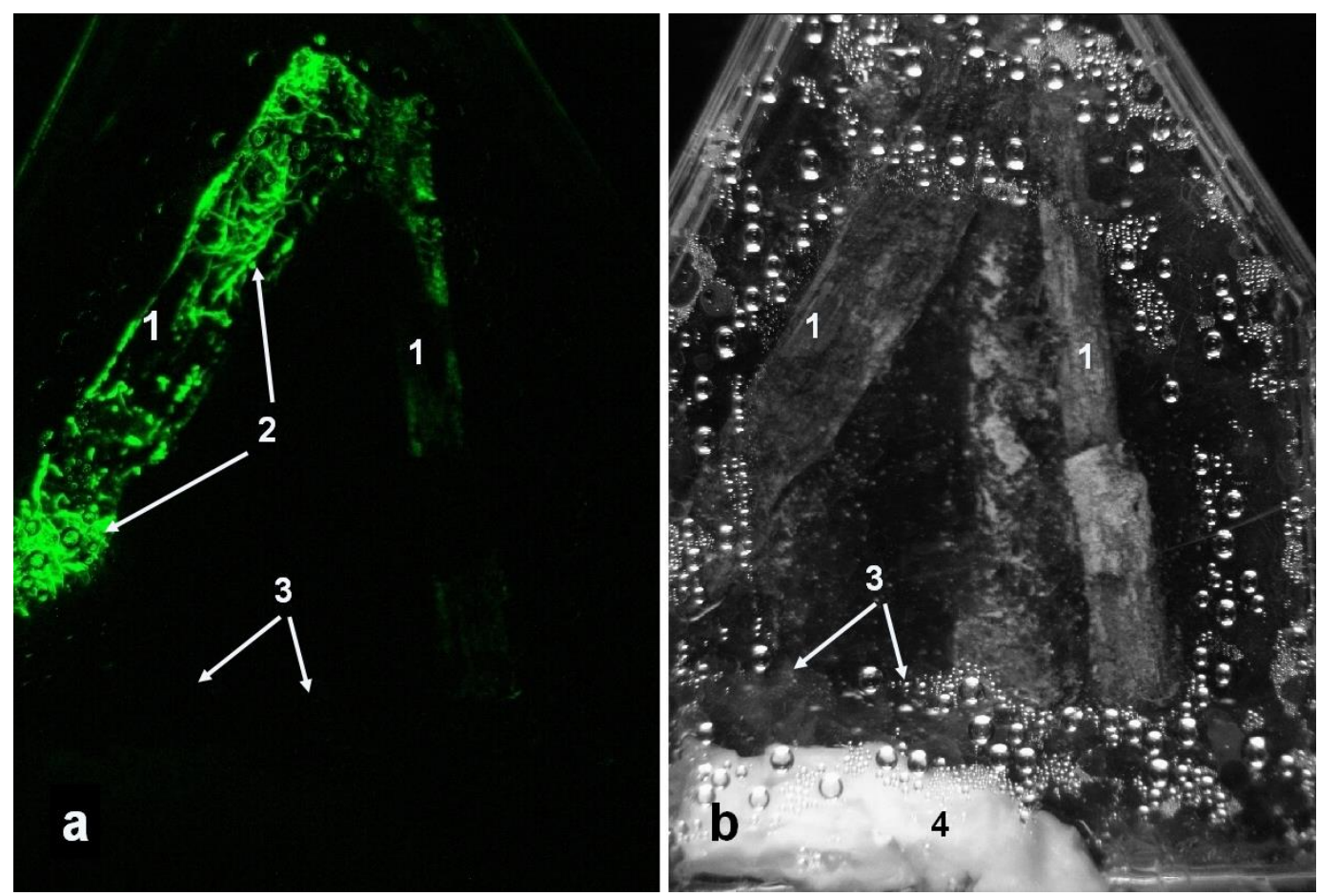

Fig. 1 - Luminescence in the dark (a) and an image in the visible light (b) of luminescent wood samples in the tissue culture flask: 1 - wood samples, 2 - mycelium, 3 - condensed water droplets on the upper surface of the tissue culture flask, 4 - cotton wool. The signal accumulation time $300 \mathrm{~s}$ (gamma 4.00, low 60021). Recorded in September 2014.

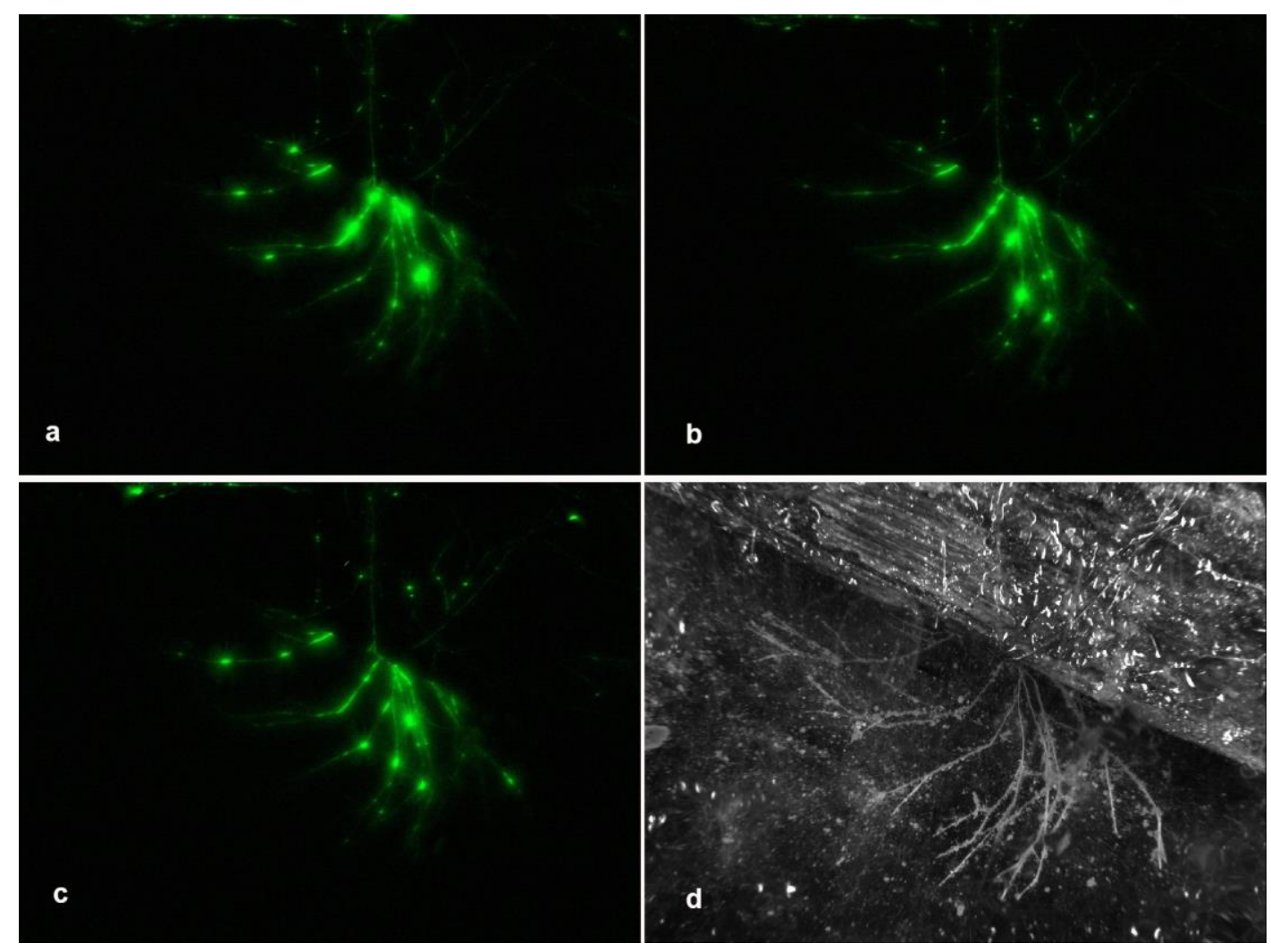

Fig. 2 - Luminescence of the surface mycelium in the tissue culture flask in the dark (a, b, c) and an image of the wood sample with aerial mycelium in the visible light (d). Luminescence recorded every $10 \mathrm{~min}$. The signal accumulation time $300 \mathrm{~s}$ (gamma 4.00, low 60021). Recorded in September 2015. 

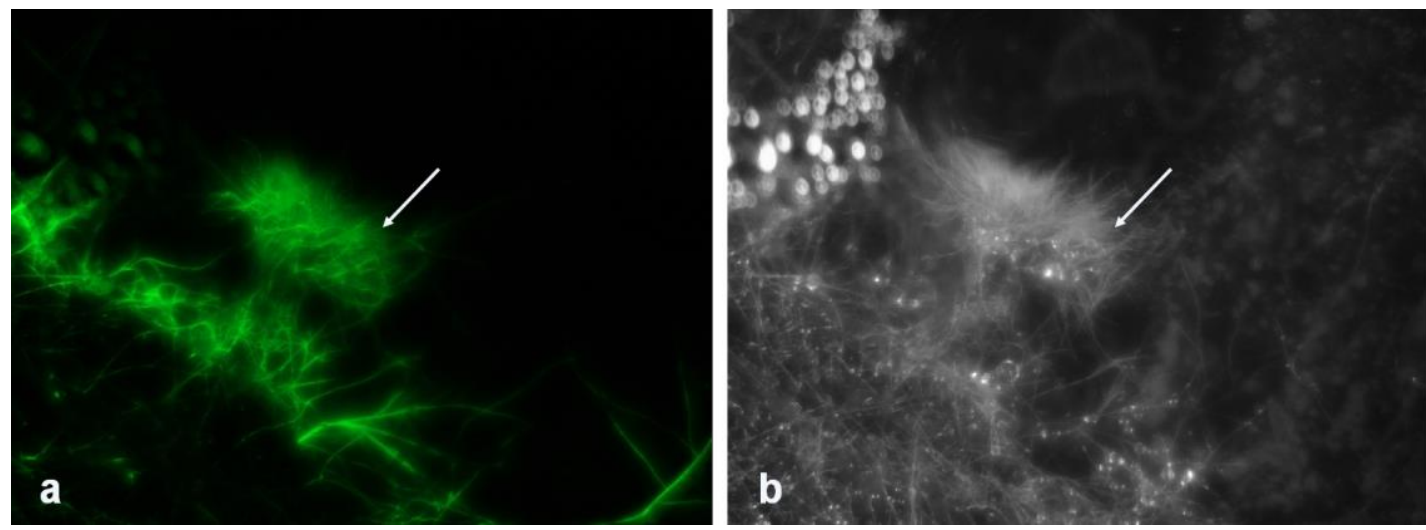

Fig. 3 - Luminescence of the aerial mycelium in the dark (a) and an image of the wood sample with aerial mycelium (shown by the arrow) and surface mycelium in the visible light (b). The signal accumulation time $300 \mathrm{~s}$ (gamma 4.00, low 60021). Recorded in February 2016.
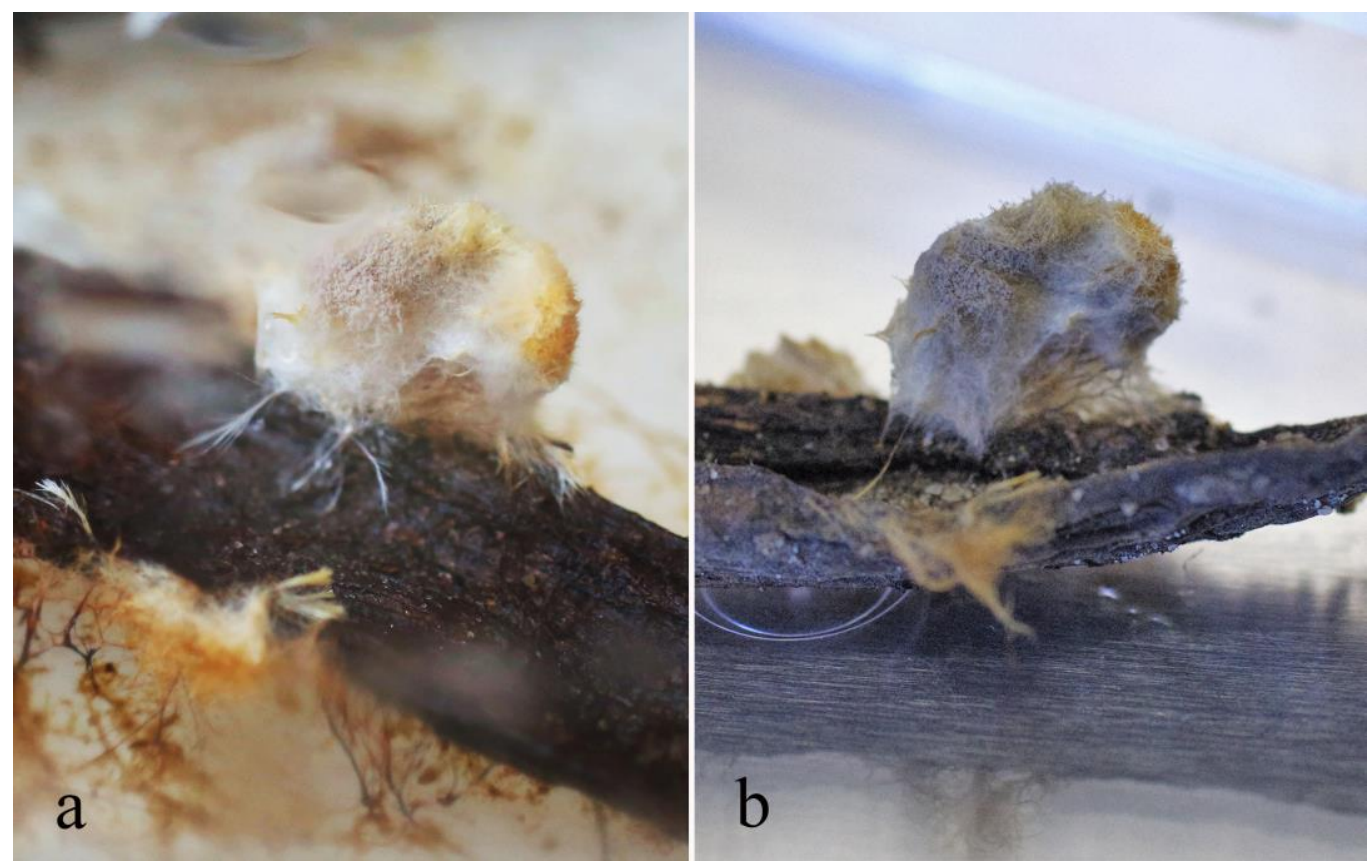

Fig. 4 - The appearance of the wood sample with the surface and aerial mycelia growing in the tissue culture flask (a) and aerial mycelium on the wood sample removed from the tissue culture flask at the end of the experiment (b). Photographs taken with a Canon EOS 6D camera.

\section{Results}

\section{Growth of mycelium on the wood during long-term storage}

Originally, wood samples placed in tissue culture flasks did not look interesting. However, after 10 months, on the surface of the wood, we detected mycelium emitting light, which was recorded with a GelDoc XR Imaging System (Fig. 1).

Then, mycelium spread over the surface of the plastic on which wood samples were sitting (Fig. 2, 3, 4a, 6a). In what follows, this mycelium will be referred to as "surface mycelium". Luminescence of the hyphae of surface mycelium was not continuous. Luminescence was recorded every 10 min, showing bright regions, which emerged and disappeared along the hyphae (Fig. 2) (Supplementary Data can be found at http://bl.ibp.ru/index.php?module=subjects\&func=viewpage \&pageid=169). 

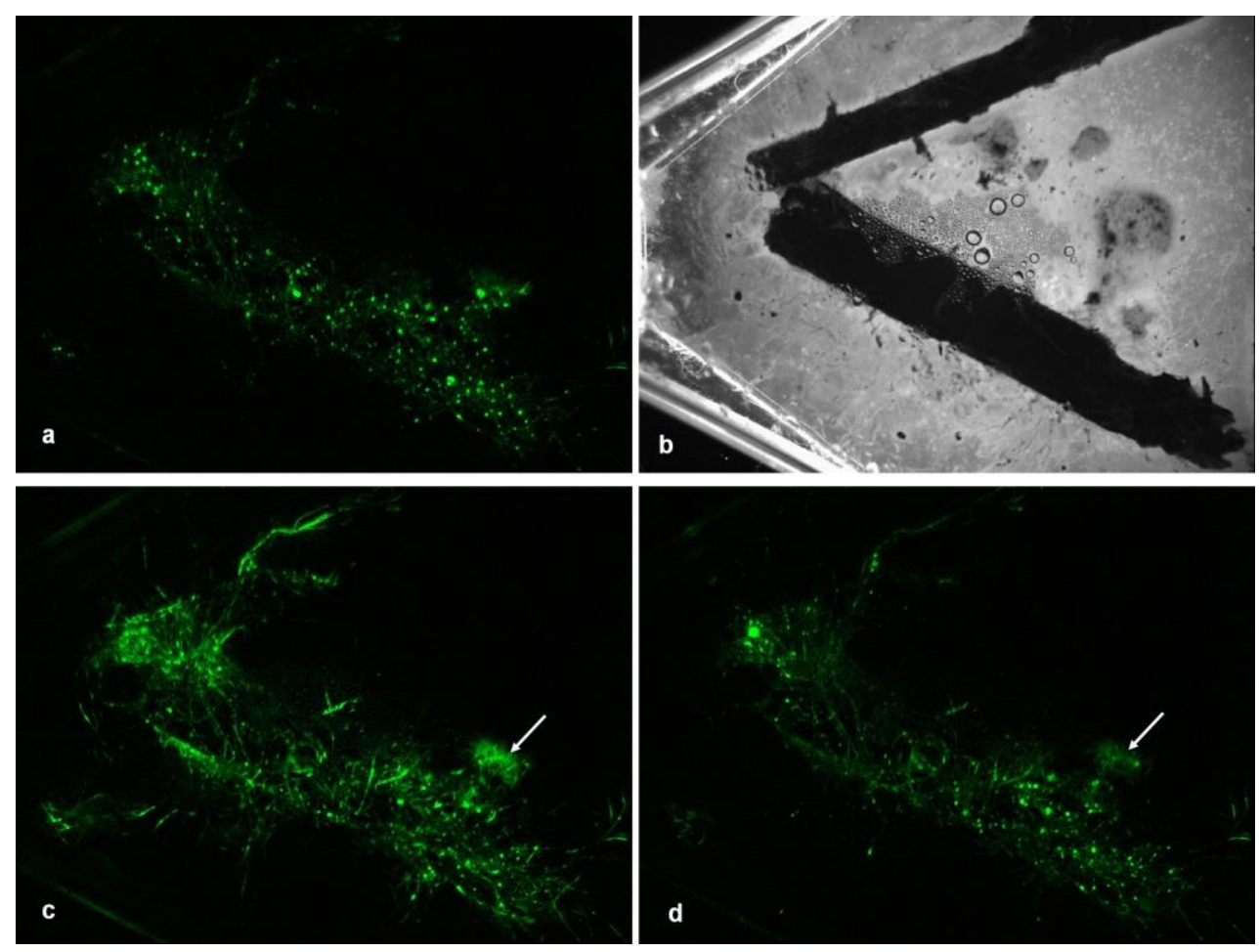

Fig. 5 - Dynamics of luminescence intensity of the mycelia after irradiation with a UV source in Universal Hood II: luminescence before exposure (a), a UV image (b), an increase in luminescence 40 min after UV exposure (c), a decrease in luminescence 60 min after UV exposure (d). The arrow points to luminescence of the local aerial mycelium formation. The signal accumulation time $300 \mathrm{~s}$ (gamma 4.00, low 60021). Recorded in February 2016.
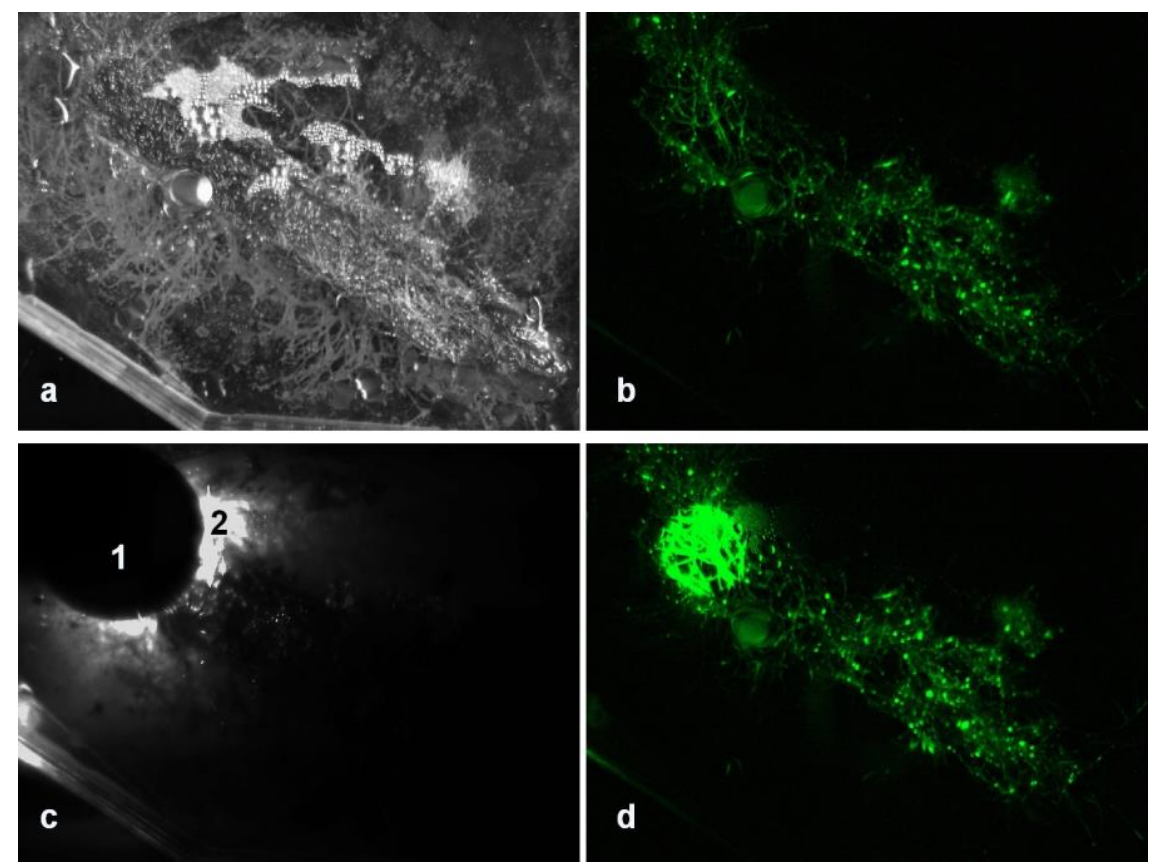

Fig. 6 - The increase in luminescence intensity of the mycelia after exposure to UV rays from the flashlight: an image in the visible light (a); luminescence of the sample before exposure (b); an image in the UV light from the flashlight (c); the increase in luminescence 6 min after UV exposure (d). Notations: 1 - the flashlight, 2 - the region exposed to ultraviolet radiation. The signal accumulation time $300 \mathrm{~s}$ (gamma 4.00, low 60021). Recorded in February 2016. 

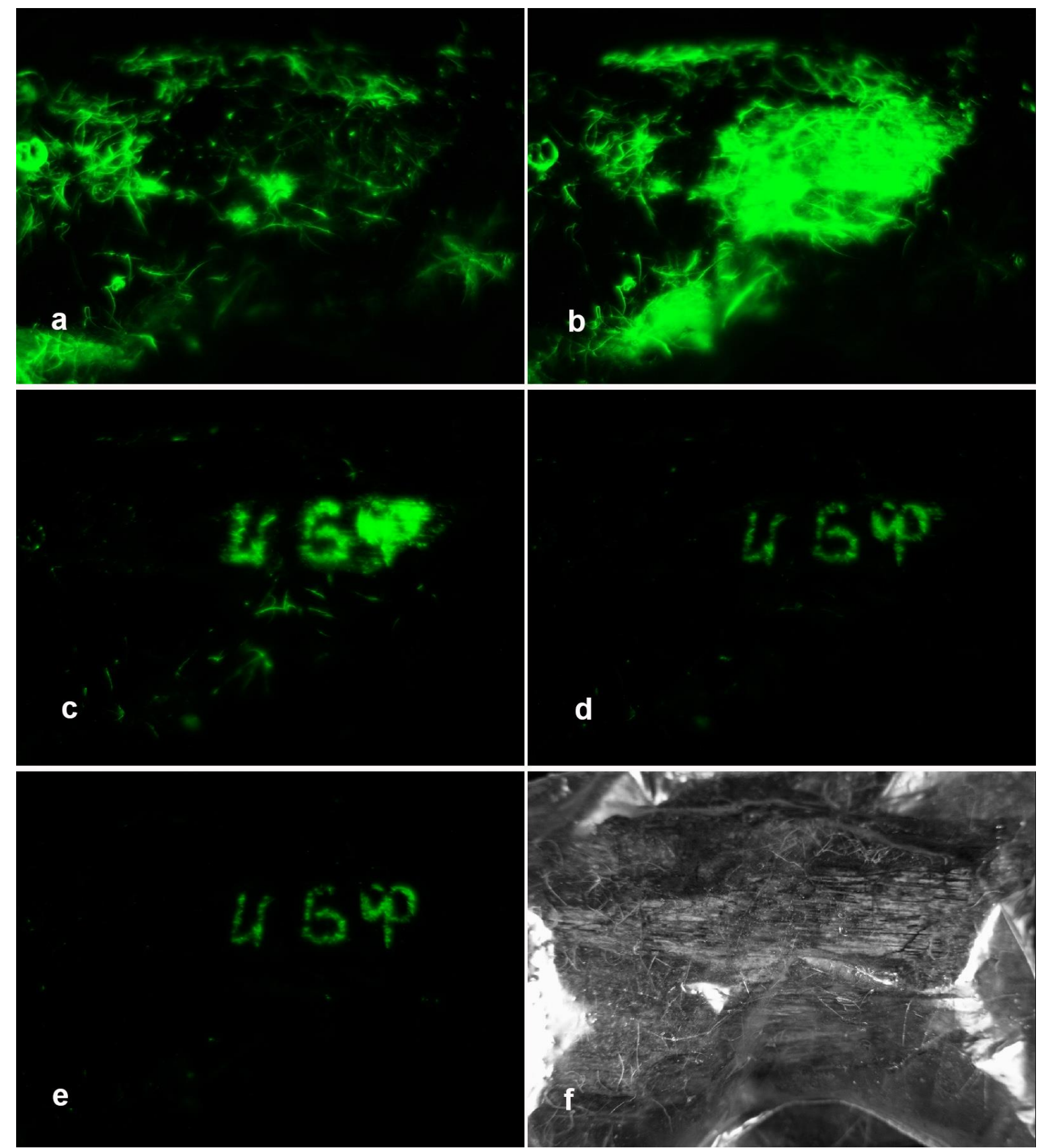

Fig. 7 - Dynamics of luminescence intensity of the mycelium after the mechanical impact on the wood sample: before the impact (a); $10 \mathrm{~min}$ after (b); $50 \mathrm{~min}$ after (c); $85 \mathrm{~min}$ after (d); $125 \mathrm{~min}$ after (e); the wood sample in the visible light (f). The signal accumulation time $300 \mathrm{~s}$. Recorded in March 2016.

After wood samples had been stored for 22 months, we detected locally growing mycelium on the surface of a sample stored in a 1300-ml tissue culture flask (Fig. 3-6). Its hyphae were tightly interlaced to produce a formation that emitted light (Fig. 3, 5, 6). This formation will be called "aerial mycelium". Aerial mycelium was originally white-colored and then it turned yellow; in five months, we observed a gray-colored region, which was growing (Fig. 4). In the gray region, mycelium lost its ability to emit light, as visualized by using a GelDoc XR Imaging System.

After 31 months of storage, as the surface and aerial mycelia of the sample lost their ability to emit light and in order to obtain a color photograph of better quality (Fig. 4) than the quality of the images obtained with the GelDoc XR Imaging System (Fig. 3, 5, 6), we opened the tissue culture flask. 

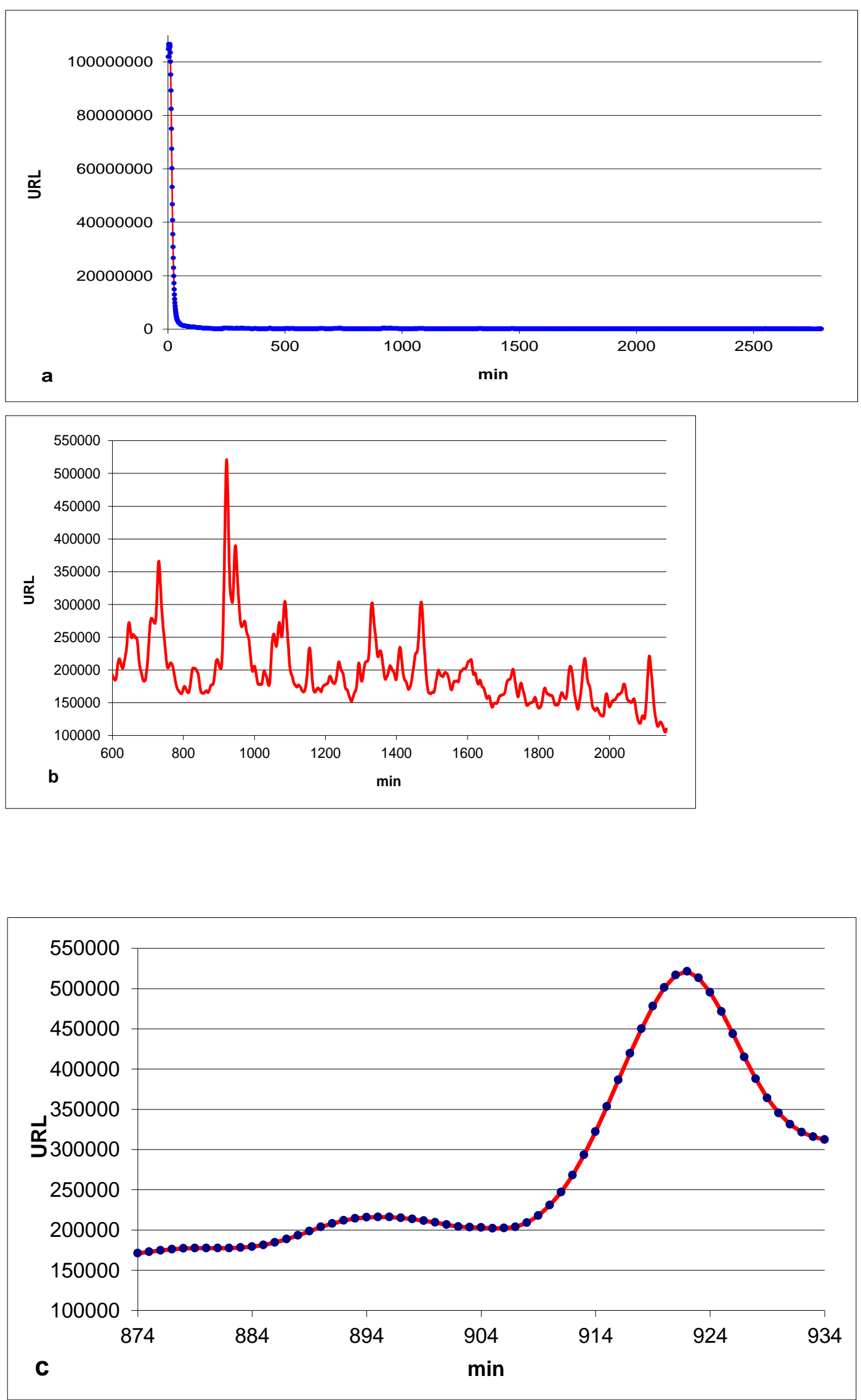

Fig. 8 -Dynamics of luminescence of the wood sample: during $2789 \mathrm{~min}(\mathrm{a}) ; 10 \mathrm{~h}$ after the sample was placed into the luminometer (measurement time of $2160 \mathrm{~min}$ ) (b); in the zone of the maximal spikes and dips (measurement time of $60 \mathrm{~min}$ ) (c). Recorded in March 2014. 
Table 1 Changes during prolonged storage wood of samples.

\begin{tabular}{lcc}
\hline \multicolumn{1}{c}{ Manipulation and the result } & Date & Storage time (months) \\
\hline $\begin{array}{l}\text { Sample collection } \\
\text { The placement of luminescent wood samples into tissue } \\
\text { culture flasks and PE bags. }\end{array}$ & $\begin{array}{c}\text { November - December 2013 } \\
\text { December } 2013\end{array}$ & 0 \\
$\begin{array}{l}\text { Growth of mycelium on wood surface in tissue culture } \\
\text { flasks, recorded with a GelDoc XR Imaging System. }\end{array}$ & September 2014 & 10 \\
$\begin{array}{l}\text { Spreading of mycelium over the surface of tissue culture } \\
\text { flasks. }\end{array}$ & January 2015 & 14 \\
$\begin{array}{l}\text { Growth of aerial mycelium on the surface of wood } \\
\text { samples }\end{array}$ & September 2015 & 22 \\
$\begin{array}{l}\text { Cessation of luminescence of mycelium on the surface of } \\
\text { tissue culture flasks, recorded with a GelDoc XR }\end{array}$ & April 2016 & 29 \\
$\begin{array}{l}\text { Imaging System. } \\
\text { Darkening of the aerial mycelium and loss of } \\
\text { luminescence. End of experiment }\end{array}$ & & 31 \\
\hline
\end{tabular}

\section{The effect of $U V$ radiation on luminescence of mycelium}

After 23 months of the storage, wood samples in tissue culture flasks were subjected to short-term UV exposure, which caused an increase in luminescence signal. The wood samples were exposed to UV rays from ultraviolet lamps in Universal Hood II located under the samples; the exposure lasted $10 \mathrm{~s}$ (Fig. 5). Local UV exposure was achieved by using an ultraviolet flashlight placed over the samples (Fig. 6).

\section{The effect of mechanical disturbance on luminescence of mycelium}

To study the effect of mechanical disturbance, we used the wood sample stored in the PE bag. Without opening the bag, we wrote on the plastic with an ordinary pencil. The pencil left no marks on polyethylene, allowing us to record the light signal from mycelium. The development of the luminescence signal is shown in Figure 7.

\section{Diurnal dynamics of mycelium luminescence}

Diurnal dynamics of luminescence was studied by using a Glomax ${ }^{\circledR}$ 20/20 luminometer. Luminescence of the wood sample in the PE bag was monitored continuously, with 1 min intervals, for over 2 days (2789 min) (Fig. 8a). Figure $8 \mathrm{~b}$ is a 24-h fragment of the recorded signals after 600min measurements, and Figure $8 \mathrm{c}$ demonstrates luminescence variations during $60 \mathrm{~min}$ in the maximum signal zone shown in Figure $8 \mathrm{~b}$.

\section{Discussion}

\section{Growth of mycelium on the wood during long-term storage}

History of changes during long-term storage of luminescent wood samples is shown in the table 1.

Results of the study described above suggest an amazing ability of the strain IBSO 2371 to remain luminescent for 31 months, with wood samples stored in hermetically sealed containers under non sterile moist conditions in laboratory. These results are consistent with the data reported by other authors, suggesting that wood retains its ability to emit light for long time periods (Sabharwal et al. 1983, Deheyn \& Latz 2007). We assume that the ability of mycelium to grow in moist non sterile medium over extended periods of time may be due to release of metabolites that inhibit the growth of other microorganisms and the nutrient-poor substrate, which cannot support the growth of bacteria or lower fungi. This assumption is corroborated by the failed attempts to derive pure culture from the surface and aerial mycelia growing on the wood: bacterial colonies proliferated on culture media on Petri dishes. 
We observed the growth of two types of mycelium capable of luminescence. The so-called surface mycelium (Fig. 1-3, 4a, 6a) developed on the surface of the wood sample, and then it was observed on the bottom of tissue culture flasks and inside the PE bags. The length of the hyphae of this mycelium could reach $55 \div 60 \mathrm{~mm}$. At a certain time, surface mycelium could develop spherical swellings (Puzyr et al. 2016). The mycelium showed characteristic non-uniform "flickering" luminescence along the hyphae (Fig. 2). This is clearly seen in the images taken every 10 min and in the Supplementary Data that can be found at http://bl.ibp.ru/index.php?module=subjects\&func=viewpage\&pageid $=169$, suggesting that this effect is an attribute of the fungus. Moreover, this effect was described in a study of the pure culture of this strain grown on agar medium (Puzyr et al. 2016).

After 22 months of storage, we detected another type of mycelium, which we called aerial mycelium (Fig. 3-6). It grew perpendicular to the wood surface, occupied a limited space, emitted light (Fig. 3-5), and consisted of tightly interlaced hyphae (Fig. 4). When the samples in tissue culture flasks lost their luminescence ability, we stopped observing them. However, the samples in $\mathrm{PE}$ bags remain luminescent, and we intend to use them in our future studies.

\section{The effect of $U V$ radiation on luminescence of mycelium}

Results of our previous studies of luminescent basidiomycetes suggest that fungal mycelium usually responds to the impact of external factors by increasing luminescence. In this study, we conducted two experiments, which proved that the strain IBSO 2371 was not an exception to this general rule. External factors used in these experiments were ultraviolet radiation and mechanical disturbance.

All experiments for the study of the effects of external factors on luminescence consisted of the procedures similar to those conducted in the investigation of the effect of UV light. First, using the GelDoc XR Imaging System, we estimated the luminescence area and intensity without any effect of the external factor (Fig. 5a). Then, the sample was exposed to UV radiation (Fig. 5b), and we recorded luminescence after the exposure (Fig. 5c, d).

Previous major studies of the effect of light on luminescence were performed with the mycelia of Armillaria and Panellus stipticus. Exposure to UV light and fluorescent light was found to inhibit bioluminescence (Airth \& Foerster 1960, Berliner 1963, Bermudes et al 1990, Weitz et al 2001). In a more recent study, Mihail and Bruhn (2007) showed that variations in luminescence were determined by the exposure time and the wavelength of the fluorescent light and by the species of Armillaria tested. It was also reported that application of X-rays did not change the luminescence emission spectrum of Neonothopanus nambi mycelium, but after 20 min of X-ray irradiation, luminescence of mycelium increased by approximately five times (Kobzeva et al 2014).

In our experiments, regardless of the UV source used (the Universal Hood II lamps or an ultraviolet flashlight), exposure of the wood sample to UV radiation caused an increase in luminescence of mycelium (Fig. 5,6). Under natural conditions, the Sun's ultraviolet radiation may serve as a "timer" of the diurnal rhythm of the fungus. Excitation seemed to be transmitted to the mycelium regions that had not been exposed to the external factor. The ultraviolet lamps contained in Universal Hood II illuminated only the lower part of the wood and the surface mycelium located on the bottom of the tissue culture flask (Fig. 5b). However, mycelium growing on the upper side of the wood also showed an increase in luminescence (Fig. 5c). Local exposure to ultraviolet radiation from the flashlight produced a similar effect. The region directly exposed to ultraviolet light looked like a bright luminescent ring. At the same time, both surface and aerial mycelia growing on the other parts of the sample also showed increased levels of luminescence (Fig. 6d).

\section{The effect of mechanical disturbance on luminescence of mycelium}

The effect of mechanical disturbance on luminescence of mycelium of the fungi growing on agar nutrient media was reported in studies by Mihail \& Bruhn (2007), Medvedeva et al. (2014), and Mihail (2015). In most experiments, luminescence was increased, and the difference from the initial level varied depending on the species of the fungus. In this study, we investigated the effect 
of mechanical disturbance of mycelium on the wood sample and transmission of excitation to other parts of mycelium, leading to an increase in luminescence (Fig. 7). On the PE bag containing a sample of light-emitting wood, we wrote three Russian letters, ИБФ (the Russian abbreviation for the Institute of Biophysics). The pencil lead pressed to the PE surface during writing did not leave any marks on the bag but impressed these letters on the mycelium on the wood sample inside the bag. The local effect was transmitted to the other parts of the mycelium and caused a response - an increase in luminescence over a vast area (Fig. 7 b). After some time, luminescence of the parts of the sample that were not directly affected by mechanical disturbance had decreased, and the letters became clearly visible (Fig. $7 \mathrm{c}$-e). This experiment shows that mycelium responds to mechanical disturbance in a few minutes, and this effect can last for several hours. The duration of the response of the fungus studied here is comparable with the duration of the response of the fungus Neonothopanus nambi (Medvedeva et al. 2014). How the local excitation caused by an external factor is transmitted to other parts of mycelium, which have not been directly affected by it but still increase their luminescence, remains an enigma for us.

\section{Diurnal dynamics of mycelium luminescence}

The GelDoc XR Imaging System enables visualization of the regions emitting light with the high level of the light signal and qualitative estimation of luminescence intensity, but it does not enable quantification of luminescence. The Glomax ${ }^{\circledR}$ 20/20 luminometer enables determination of the range within which the light flux varies (Fig. 8). When the PE bag with the sample is removed from the thermostatic chamber and placed into the luminometer, mycelium is subjected to mechanical disturbance, which causes strong emission of light energy (Fig. 8a). Therefore, in order to obtain accurate diurnal dynamics of luminescence, the sample must stay inside the luminometer for a long time. Other external factors, such as temperature, must remain constant too, as they also influence the level of luminescence. The high original level of the signal emitted by the sample placed into the luminometer (Fig. 8a) does not allow detecting the subsequent variations in luminescence. This can be done, however, by analyzing the data on the luminescence signal intensity from certain regions of diurnal dynamics (Fig. 8 b, c). Variations in the luminescence signal observed after the first $10 \mathrm{~h}(600 \mathrm{~min}$ ) for the following $2160 \mathrm{~min}$ (Fig. $8 \mathrm{~b}$ ) suggest that none of the variations in luminescence can be related to diurnal dynamics, and light flashes occur irregularly. At the same time, the "basic" intensity of the signal tends to decrease, suggesting that the fungus has not returned to its initial state after perturbations caused by its placement into the luminometer. The greatest difference between the values observed for the disturbed and undisturbed mycelium may be more than 8000-fold (Fig. 8a), and the spikes and dips of the signal emitted by the undisturbed mycelium after $10 \mathrm{~h}$ of measurements may differ by a factor of 3 , these variations sometimes occurring within one hour (Fig. 8 c). Our results are in good agreement with the data reported by Mihail (2013). She wrote that luminescence was not stable, and that peaks and dips were recorded. It was assumed that the transient stimuli influenced the luminescence intensity.

Our results are also consistent with the observations described by Weitz et al. (2001) and Deheyn \& Latz. (2007), suggesting that light production does not vary between day and night. At the same time, the presence of spikes and dips is indicative of the difference between our results and the data reported by Weitz et al. (2001), who stated that light production in the fungus is a continuous phenomenon. A significant difference is that our results do not suggest any diurnal periodicity, which is described in studies by other authors (Berliner 1961, Kamzolkina 1982, Bermudes et al. 1990, Oliveira et al. 2015).

The morphological traits of the strain IBSO 2371 are characteristic of basidiomycetes (the structure of the mycelial colony, hyphal buckling, etc.). However, based on the ITS sequencing data, we failed to identify the culture by searching for homologous sequences in Gen Bank (NCBI). The closest sequences belong to several unidentified "uncultured mycorrhizal fungi" (Puzyr et al. 2015). No fruiting bodies of this fungus have been found in nature, making its identification even more difficult. We assume that the variable level of luminescence, in combination with other properties described above, is an individual trait of this fungus species. Thus, results obtained in 
this study suggest that luminescent wood found on Borneo Island contains mycelium of a fungus species, whose luminescent properties are essentially different from those of the fungi described in the scientific literature.

\section{Acknowledgements}

This study was supported by the state budget allocated to the fundamental research at the Russian Academy of Sciences (project no. 0360-2014-0006) and Program No.II.2 «Integration and Development» of the Siberian Branch of the Russian Academy of Sciences (project no. 03602015-0003).The authors are grateful to Dr. N.V. Psurtseva (V.L. Komarov Botanical Institute RAS, Russia) for conducting the genetic investigation of the mycelium.

\section{References}

Airth RL, Foerster GE. 1960 - Some aspects of fungal bioluminescence. J Cell Compar Physiol 56, 173-182.

Airth RL, Foerster GE. 1962 - The isolation of catalytic components required for cell-free fungal bioluminescence. Arch Biochem Biophys 97, 567-573.

Airth, RL, Foerster GE, Behrens QP. 1966 - The Luminous Fungi. In Bioluminescence in progress. (Edited by F. H. Johnson and Y. Haneda), Princeton University Press. pp. 203-233.

Berliner MD. 1961 - Diurnal periodicity of luminescence in three basidiomycetes. Science 134, 739-740.

Berliner MD. 1963 - The action of monochromatic ultraviolet radiation on luminescence in Armillaria mellea. Rad Res 19: 392-401.

Bermudes D, Gerlach VL, Nealson KH. 1990 - Effects of culture conditions on mycelial growth and luminescence in Panellus stypticus. Mycologia 82, 295-305.

Bilai VI. 1982 - Methods of experimental mycology. Naukova Dumka, 550 pages.

Bondar VS, Rodicheva EK, Medvedeva SE, Tyulkova NA, Tyaglik AB, Shpak BA, Gitelson JI. 2013 - On the mechanism of luminescence of the fungus Neonothopanus nambi. Doklady Biochemistry and Biophysics 449, 80-83.

Bondar VS, Shimomura O, Gitelson JI. 2012 - Luminescence of higher mushrooms. Journal of Siberian Federal University. Biology 5(4), 331-351.

Capelari M, Desjardin DE, Perry BA, Asai T, Stevani CV. 2011 - Neonothopanus gardneri: a new combination for a bioluminescent agaric from Brazil. Mycologia 103, 1433-1440.

Chew AL, Tan YS, Desjardin DE, Musa MY, Sabaratnam V. 2014 - Four new bioluminescent taxa of Mycena sect. Calodontes from Peninsular Malaysia. Mycologia 106(5), 976-88. doi: 10.3852/13-274.

Deheyn DD, Latz MI. 2007 - Bioluminescence characteristics of a tropical terrestrial fungus (Basidiomycetes). Luminescence 22, 462-467.

Desjardin DE, Perry BA, Lodge DJ, Stevani CV, Nagasawa E. 2010 - Luminescent Mycena: new and noteworthy species. Mycologia, 102(2), 459-477. doi: 10.3852/09-197.

Horswell J, Weitz H, Percival H, Speir T. 2006 - Impact of heavy metal amended sewage sludge on forest soils as assessed by bacterial and fungal biosensors. Biol Fert Soils 42, 569-576.

Kamzolkina OV. 1982 - Oscillation of bioluminescence in submerged culture of Armillaria mellea (Fr) Kumm. Mikol Fitopatol 16, 323-325.

Kobzeva TV, Melnikov AR, Karogodina TY, Zikirin SB, Stass DV, Molin YN, Rodicheva EK, Medvedeva SE, Puzyr AP, Burov AA, Bondar VS, Gitelson JI. 2014 - Stimulation of luminescence of mycelium of luminous fungus Neonothopanus nambi by ionizing radiation. Luminescence 29, 703-710.

Medvedeva SE, Artemenko KS, Krivosheenko AA, Rusinova AG, Rodicheva EK, Puzyr AP, Bondar VS. 2014 - Growth and light emission of luminous basidiomycetes cultivated on solid media and in submerged culture. Mycosphere 5, 565-577. doi 10.5943/mycosphere/5/4/9. 
Mendes LF, Bastos EL, Desjardin D E, Stevani CV. 2008 - Influence of culture conditions on mycelial growth and bioluminescence of Gerronema viridilucens. FEMS Microbiol Lett 282, 132-139.

Mendes LF, Stevani CV. 2010 - Evaluation of metal toxicity by a modified method based on the fungus Gerronema viridilucens bioluminescence in agar medium. Environ Toxicol Chem 29, 320-326.

Mihail JD, Bruhn JN. 2007 - Dynamics of bioluminescence by Armillaria gallica, A. mellea and A. tabescens. Mycologia 99(3), 341-50.

Mihail JD. 2013 - Comparative bioluminescence dynamics among multiple Armillaria gallica, A. mellea and A. tabescens genets. Fungal Biol 117, 202-210.

Mihail JD. 2015 - Bioluminescence patterns among North American Armillaria species. Fungal Biol 119(6), 528-537. doi: 10.1016/j.funbio.2015.02.004.

Mori K, Kojima S, Maki S, Hirano T, Niwa H. 2011 - Bioluminescence characteristics of the fruiting body of Mycena chlorophos. Luminescence 26, 604-610.

Oliveira AG, Desjardin DE, Perry BA, Stevani CV. 2012 - Evidence that a single bioluminescent system is shared by all known bioluminescent fungal lineages. Photochem Photobiol Sci 11, $848-852$.

Oliveira AG, Stevani CV, Waldenmaier HE, Viviani V, Emerson JM, Loros JJ, Dunlap JC. 2015 Circadian control sheds light on fungal bioluminescence. Curr Biol 25(7), 964-968.

Oliveira AG, Stevani CV. 2009 - The enzymatic nature of fungal bioluminescence. Photochem Photobiol Sci 8, 1416-1421.

Paton GI, Viventsova E, Kumpene J, Wilson MJ, Weitz HJ, Dawson JJC. 2006 - An ecotoxicity assessment of contaminated forest soils from the Kola Peninsula. Sci Total Environ 355, 106-117.

Purtov KV, Petushkov VN, Baranov MS, Mineev KS, Rodionova NS, Kaskova ZM, Tsarkova AS, Petunin AI, Bondar VS, Rodicheva EK, Medvedeva SE, Oba Y, Oba Y, Arseniev AS, Lukyanov S, Gitelson JI, Yampolsky IV. 2015 - The Chemical Basis of Fungal Bioluminescence. Angew Chem Int Ed 54, 8124-8128.

Puzyr AP, Medvedeva SE, Bondar VS. 2016 - The use of glowing wood as a source of luminescent culture of fungus mycelium. Mycosphere 7(1), 13-29. doi 10.5943/mycosphere/7/1/2.

Puzyr AP, Medvedeva SE, Psurtseva NV. 2015 - Glowing wood - a source for culture of new higher mushrooms. The $5^{\text {th }}$ Congress of Biophysicists of Russia. Rostov-on-Don Proceedings V. 2, p. 178.

Sabharwal SC, Kathuria SP, Dhingra MM. 1983 - A new bioluminescent fungal system. Journal of Biosciences. 5(1), 53-62.

Vydriakova GA, Gusev AA, Medvedeva SE. 2011 - Effect of organic and inorganic toxic compounds on luminescence of luminous fungi. Prikl Biokhim Mikrobiol 47(3), 324-329. Russian.

Weitz HJ, Ballard AL, Campbell CD, Killham K. 2001 - The effect of culture conditions on the mycelial growth and luminescence of naturally bioluminescent fungi. FEMS Microbiol Lett 202, 165-170.

Weitz HJ, Ballard AL, Campbell CD, Killham K. 2002 - Development of a novel, bioluminescence-based, fungal bioassay for toxicity testing. Environ Microbiol 4, 422-429.

http://bl.ibp.ru/index.php? module=subjects $\&$ func=viewpage $\&$ pageid=169 